\title{
ANL/PHy/CP-102364
}

The submitted manuscript has been created by the University of Chicago as Operator of Argonne National Laboratory ("Argonne") under Contract No. W-31-109-ENG-38 with the U.S. Department of Energy. The U.S. Government retains for itself, and others acting on its behalf, a paid-up, nonexclusive, irrevocable worldwide license in said article to reproduce, prepare derivative works, dis tribute copies to the public, and perform publicly and display publicly, by or on behalf of the Government.

\section{First Results From the PHOBOS Experiment at RHIC}

A. H. Wuosmaa ${ }^{1}$, for the PHOBOS Collaboration: B. B. Back ${ }^{1}$, M. D. Baker ${ }^{2}$, D. S. Barton ${ }^{2}$, S. Basilev, , B. D. Bates ${ }^{5}$, R. Baum ${ }^{8}$, R. R. Betts ${ }^{1,7}$, A. Białas ${ }^{4}$, R. Bindel $^{8}$, W. Bogucki ${ }^{3}$, A. Budzanowski ${ }^{3}$,W. Busza ${ }^{5}$, A. Carroll ${ }^{2}$, M. Ceglia ${ }^{2}$, Y.-H.Chang ${ }^{6}$, A. E. Chen ${ }^{6}$, T. Coghen ${ }^{3}$, C. Conner ${ }^{7}$, W. Czyz ${ }^{4}$, B. Dabrowski ${ }^{3}$, M. P. Decowski ${ }^{5}$, M. Despet ${ }^{3}$, P. Fita ${ }^{5}$, J. Fitch ${ }^{5}$, M. Friedl ${ }^{5}$, K. Galuska ${ }^{3}$, R. Ganz $^{7}$, E. Garcia ${ }^{8}$, N. George ${ }^{1}$, J. Godlewski ${ }^{3}$, C. Gomes ${ }^{5}$, E. Griesmayer ${ }^{5}$, K. Gulbrandsen $^{5}$, S. Gushue ${ }^{2}$, J. Halik ${ }^{3}$, C. Halliwell ${ }^{7}$, P. Haridas ${ }^{5}$, A. Hayes ${ }^{9}$, G. A. Heintzelman ${ }^{2}$, C. Henderson ${ }^{5}$, R. Hollis ${ }^{7}$, R. Hołynski ${ }^{3}$, B. Holzman ${ }^{7}$, E. Johnson $^{9}$, J. Kane ${ }^{5}$, J. Katzy ${ }^{5,7}$, W. Kita ${ }^{3}$, J. Kotuła ${ }^{3}$, H. Kraner ${ }^{2}$, W. Kucewicz ${ }^{7}$, P. Kulinich , C. Law ${ }^{5}$, M. Lemler ${ }^{3}$, J. Ligocki ${ }^{3}$, W. T. Lin ${ }^{6}$, S. Manly ${ }^{9}, 10$, D. McCleod $^{7}$, J. Michałowski ${ }^{3}$, A. Mignerey ${ }^{8}$, J. Mülmenstädt ${ }^{5}$, M. Neal ${ }^{5}$, R. Nouicer $^{7}$, A. Olszewski ${ }^{2,3}$, R. Pak ${ }^{2}$, I. C. Park ${ }^{9}$, M. Patel ${ }^{5}$, H. Pernegger ${ }^{5}$, M. Plesko $^{5}$, C. Reed ${ }^{5}$, L. P. Remsberg ${ }^{2}$, M. Reuter ${ }^{7}$, C. Roland ${ }^{5}$, G. Roland ${ }^{5}$, D. Ross $^{5}$, L. Rosenberg ${ }^{5}$, J. Ryan ${ }^{5}$, A. Sanzgiri ${ }^{10}$, P. Sarin ${ }^{5}$, P. Sawicki ${ }^{3}$, J. Scaduto $^{2}$, J. Shea ${ }^{8}$, J. Sinacore ${ }^{2}$, W. Skulski ${ }^{9}$, S. G. Steadman ${ }^{5}$, G. S. F. Stephans $^{5}$, P. Steinberg ${ }^{2}$, A. Straczek ${ }^{3}$, M. Stodulski ${ }^{3}$, M. Strek ${ }^{3}$, Z. Stopa ${ }^{3}$, A.

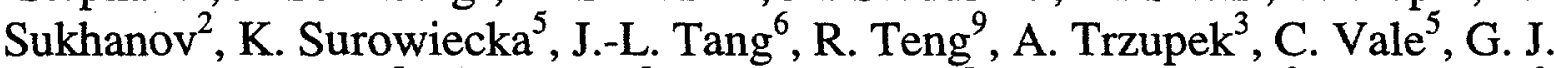
van Nieuwenhuizen ${ }^{5}$, R. Verdier ${ }^{5}$, B. Wadsworth ${ }^{5}$, F. L. H. Wolfs ${ }^{9}$, B. Wosiek ${ }^{3}$, K. Wozniak ${ }^{3}$, A. H. Wuosmaa ${ }^{1}$, B. Wysłouch ${ }^{5}$, K. Zalewski ${ }^{4}$, P. Zychowski ${ }^{3}$

\author{
${ }^{1}$ Physics Division, Argonne National Laboratory, Argonne IL, 60439-4843 \\ ${ }^{2}$ Chemistry and C-A Departments, Brookhaven National Laboratory, Upton NY 11973-5000 \\ ${ }^{3}$ Institute of Nuclear Physics, Krakow, Poland \\ ${ }^{4}$ Department of Physics, Jagellonian University Krakow, Poland \\ ${ }^{5}$ Laboratory for Nuclear Science, Massachusetts Institute of Technology, Cambridge MA 02139-4307 \\ ${ }^{6}$ Department of Physics, National Central University, Chung-Li, Taiwan \\ ${ }^{7}$ Department of Physics, University of Illinois at Chicago, Chicago IL 60607-7059 \\ ${ }^{8}$ Department of Chemistry, University of Maryland, College Park MD 20742 \\ ${ }^{9}$ Deparment of Physics and Astronomy, University of Rochester, Rochester NY 14627 \\ ${ }^{10}$ Department of Physics, Yale University, New Haven CT 06520
}

\begin{abstract}
The PHOBOS experiment at RHIC has measured the charged-particle density $d N / d \eta$ at mid-rapidity for cedntral Au+Au collisions at center of mass energies of $\sqrt{s_{N N}}=56$, and $130 \mathrm{GeV}$. We deduce that $d N / d \eta=408 \pm 12$ (stat) \pm 30 (syst) and $555 \pm 12$ (stat) \pm 35 (syst) for collision energies of 56 , and $130, \mathrm{GeV}$, respectively. These numbers suggest energy densities that are some $70 \%$ higher than have been achieved in any heavy-ion collisions previously studied, and also $25-40 \%$ higher than nucleon-nucleon collisions at comparable center of mass energies.
\end{abstract}




\section{DISCLAIMER}

This report was prepared as an account of work sponsored by an agency of the United States Government. Neither the United States Government nor any agency thereof, nor any of their employees, make any warranty, express or implied, or assumes any legal liability or responsibility for the accuracy, completeness, or usefulness of any information, apparatus, product, or process disclosed, or represents that its use would not infringe privately owned rights. Reference herein to any specific commercial product, process, or service by trade name, trademark, manufacturer, or otherwise does not necessarily constitute or imply its endorsement, recommendation, or favoring by the United States Government or any agency thereof. The views and opinions of authors expressed herein do not necessarily state or reflect those of the United States Government or any agency thereof. 


\section{DISCLAIMER}

Portions of this document may be illegible in electronic image products. Images are produced from the best available original document. 


\section{INTRODUCTION}

In June of 2000, the Relativistic Heavy Ion Collider (RHIC) produced its first collisions of $\mathrm{Au}$ ions at energies of $\sqrt{s_{N N}}=56$, and $130 \mathrm{GeV}$, the highest energies at which heavy ions have yet been collided in the laboratory. Of particular interest are global characteristics of the collisions that provide information about the thermodynamic properties of the early evolutionary phases of the expanding hot system. Chief among these are the values of the energy, and entropy density a few $\mathrm{fm} / \mathrm{c}$ after the collision. Bjorken ${ }^{1}$ has suggested that these quantities may be directly related to the density of charged particles emitted at mid rapidity. In order to probe these conditions, it is therefore interesting to study the charged-particle density at mid-rapidity, as well as its scaling with collision energy and the number of interacting nucleons. A comparison of the charged particle density per participant nucleon with data from proton-proton or proton-antiproton scattering may yield information about the collective effects or rescattering. The early PHOBOS measurements attempt to address these questions.

\section{EXPERIMENTAL DETAILS}

The PHOBOS experiment consists primarily of a large collection of silicon pad detectors. In the full experimental configuration used to take data in 2000 , the setup consisted of a large acceptance $(\sim 4 \pi)$ multiplicity detector, a small-acceptance two-arm silicon pad tracking spectrometer within a $2 \mathrm{~T}$ magnetic field, plastic-scintillator and Cerenkov-radiator trigger detectors, two plastic-scintillator time-of-flight walls, and two zero-degree calorimeters (ZDCs). A more detailed description of the experimental setup may be found in References 2 and 3.

In the initial phases of collider operations at RHIC, there was some, ultimately unfounded, concern about radiation damage to the silicon sensors and delicate readout electronics in PHOBOS, in the case of possible large background radiation fields. Consequently, for the first few weeks of operations a small subset of the silicon pad detectors was installed. This collection included 13 of the total 92 elements of the central multiplicity detector, six of a total of 24 of the central vertex tracking detectors, and six planes of tracking spectrometer. The plastic-scintillator trigger detectors, as well as the ZDCs were also installed. In addition, the magnet was not energized for this commissioning period. A schematic diagram of the experimental setup for this initial stage appears in Fig. 1.

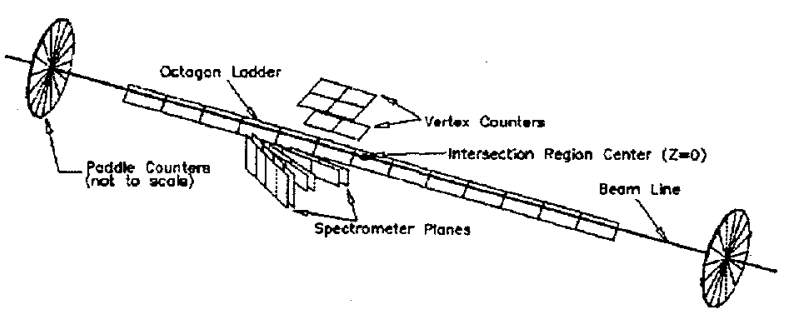

FIGURE 1. Schematic diagram of the commissioning run setup.

\section{DATA ANALYSIS}

The first step in data analysis concerns the identification of collision events. This identification was achieved using timing data from the PHOBOS trigger detectors. Particles produced by collisions in the nominal interaction region at the center of the experiment were detected in the two plastic scintillator trigger arrays. The difference in time-of-arrival for these particles at the trigger counters was approximately zero, whereas events produced by background interactions upstream of the experiment traversed one, then the other trigger array, yielding very different timing characteristics. This timing measurement provided a very clean separation between collisions and background. The contribution to the sample of collision data from double beam-gas interactions was calculated from the observed rate of single beam-gas interactions, and was found to be negligible.

The centrality of the collision events was determined from a measurement of the total energy deposited in the two paddle-counter arrays (see Fig. 1). To avoid contributions to this energy signal from lowenergy particles that are not minimum ionizing, and consequently deposit more energy, the paddles with the four highest of the 16 energy signals were taken out of the analysis, and the remaining data were used to determine the centrality. Figure 2 shows the resulting paddle energy distribution obtained at a center-of-mass collision energy of $130 \mathrm{GeV}$. The shaded region denotes the $6 \%$ of events that deposit 
the most energy. These are the most central collisions included in this analysis.

To determine the number of participating nucleons for this event sample, events from the HIJING event generator ${ }^{4,5}$ were processed with a detailed simulation of the response of the trigger counters using the GEANT simulation package. The same $6 \%$ energy cut was applied to the HIJING Monte-Carlo data, and the corresponding number of participating nucleons from HIJING was determined to be $<N_{\text {part }}>=330 \pm 4($ stat $) \pm \pm_{15}^{10}$ (syst) and $\left\langle N_{p a n t}\right\rangle=343 \pm 4($ stat $) \pm_{14}^{7}$ (syst) at $\sqrt{s_{N N}}=56$, and $130 \mathrm{GeV}$, respectively.

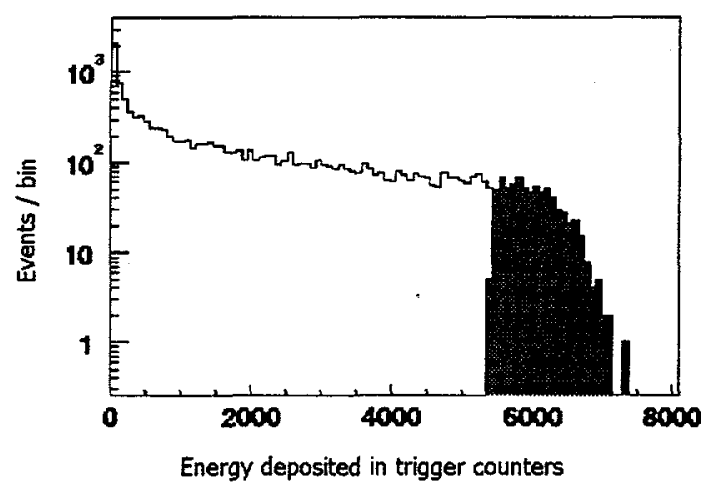

FIGURE 2. Energy spectrum measured in the trigger counters. The shaded region approximately corresponds to the $6 \%$ most central collisions.

In order to determine the pseudorapidity $\eta$ for each particle, it is first necessary to identify the collision vertex. In PHOBOS this may be accomplished in a number of different ways. For the analysis presented here, vertices were identified using found tracks in the silicon tracking spectrometer. Here, tracks are identified with a road-following algorithm connecting hits in at least four of the six silicon spectrometer planes. The resulting tracks are then analyzed to determine if they project back to the same point in space to within approximately $1 \mathrm{~mm}$. The average number of reconstructed tracks at the lower energy was approximately 13 , and at the higher energy 18 , for central collisions. Figure 3 shows a typical event obtained at a center of mass energy of $\sqrt{s_{N N}}=56 \mathrm{GeV}$.

After identification of the collision vertex, the hits in the first two planes of the tracking spectrometer, as well as those in the two planes of the vertex detector, are analyzed to reconstruct "tracklets." A tracklet is a straight-line track emanating from the collision vertex, which connects to two points in first two layers of the tracking spectrometer, or the two layers of the vertex detector. As the tracklets are required to originate at the identified vertex, the number of identified tracklets is insensitive to backgrounds arising from secondary particles produced, for example, in the beam pipe.

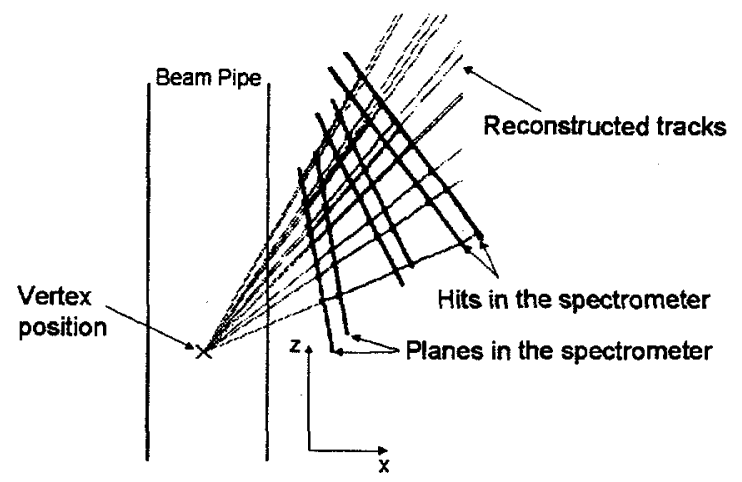

FIGURE 3. Reconstructed tracks for an Au+Au collision at $\sqrt{s_{N N}}=56 \mathrm{GeV}$. The thick lines represent planes of the tracking spectrometer, and the thin lines denote reconstructed tracks.

In order to deduce the pseudorapidity density from the measured number of tracklets, a number of corrections were applied to the data to account for the acceptance of the spectrometer and vertex detectors, inefficiencies of tracklet finding, false tracks from noise hits, as well as secondary particles and the products of weak decays of strange particles. These corrections were obtained from analyses of the response of the detector using the event generator HIJING and the simulation code GEANT. For each event, the Monte-Carlo data were analyzed in precisely the same manner as real events. The number of tracklets per event for the Monte-Carlo sample was compared to the actual number of particles emitted in to the spectrometer or vertex acceptance (for $|\eta|<1$ ), and the corresponding correction factor was then applied to the real data. This procedure was also carried out for events simulated using the VENUS event generator. The correction factors obtained from the HIJING and VENUS event sets differed by less than $5 \%$, demonstrating that this procedure produces a reliable measure of the charged-particle density.

\section{RESULTS AND DISCUSSION}

From this analysis, we obtain the values $d N /\left.d \eta\right|_{|\eta|<1}=408 \pm 12$ (stat) \pm 30 (syst) for $\sqrt{s_{N N}}=56 \mathrm{GeV}$, and $555 \pm 12$ (stat) \pm 35 (syst) at $\sqrt{s_{N N}}=130 \mathrm{GeV}$. To compare our results with those of nucleon-nucleon 
scattering at similar center of mass energies, we divide our results by the approximate number of participating nucleon-nucleon pairs $\left\langle 0.5 N_{\text {parr }}\right\rangle$. We determine $d N /\left.d \eta\right|_{|\eta|<l} /<0.5 N_{\text {part }}>=2.47 \pm 0.10$ (stat) \pm 0.25 (syst.) at $56 \mathrm{GeV}$, and $3.24 \pm 0.10$ (stat) \pm 0.25 (syst.) at 130 $\mathrm{GeV}$. Figure 4 shows the PHOBOS data points, as well as the results from the SPS $\mathrm{Pb}+\mathrm{Pb}$ collisions ${ }^{7}$ at $\sqrt{s_{N N}}=17.8 \mathrm{GeV}$, and from $p \bar{p}$ collisions from $\mathrm{CERN}^{8}$. We observe that the central pseudo-rapidity density for charged particles is approximately $70 \%$ higher at $130 \mathrm{GeV}$ than for the SPS energy, suggesting a correspondingly higher central energy density for these collisions. Also, the number of charged particles produced per participant nucleon is approximately $40 \%$ larger for $\mathrm{Au}+\mathrm{Au}$ collisions than for nucleonnucleon collisions at similar bombarding energies, ruling out simple superposition models, such as the wounded nucleon model ${ }^{9}$. Also, the increase in the normalized particle densities of approximately $30 \%$ between $\sqrt{s_{N N}}=56$ and $130 \mathrm{GeV}$ is dramatically larger than for proton-antiproton collisions.

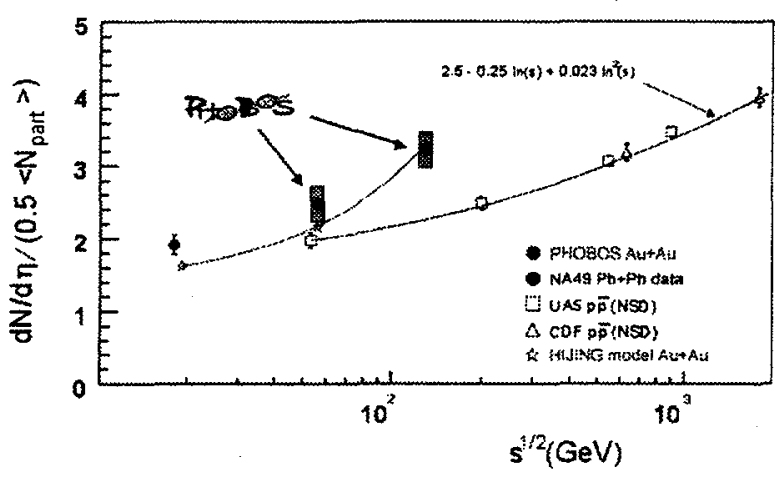

FIGURE 4. Charged particle density $d N / d \eta(|\eta|<1)$ divided by number of participant pairs, plotted versus center of mass collision energy.

Our current results are in good agreement with the predictions ${ }^{5}$ of the code HIJING, which includes the effects of particle production via hard-scattering processes, as seen in Fig. 4. This calculation also predicts the dependence of $d N / d \eta$ on $N_{\text {parn }}$, as well as on $\eta$. An experimental determination of this behavior can readily distinguish between HIJING-like models, and saturation models employing different $\mathrm{pQCD}$ cutoff schemes (e.g. Ref. 10). Also, while the effects of jet quenching in HIJING are modest at the highest energy studied so far, the results at $\sqrt{s_{N N}}=200 \mathrm{GeV}$ show a marked sensitivity to this aspect of the calculation. It will be extremely interesting to follow the evolution of the data to even higher energies.
With the conclusion of the physics run in September 2000, PHOBOS has accumulated a data set consisting of more than $3.5 \mathrm{M} \mathrm{Au}+\mathrm{Au}$ collisions at $\sqrt{s_{N N}}=130 \mathrm{GeV}$, using the fully instrumented experimental setup. These data are currently under analysis and will shed additional light on the issues examined in the early running phase.

\section{ACKNOWLEDGMENTS}

We gratefully acknowledge the support of the personnel of the RHIC facility, the C-A and Chemistry Departments at BNL. We thank Fermilab and CERN for their assistance in silicon detector assembly. This work was supported in part by U.S. Department of Energy grants DE-AC02-98CH10886, DE-FG0293ER40802, DE-FC02-94ER40818, DE-FG0294ER40865, DE-FG02-99ER41099, W-31-109-ENG38, and National Science Foundation Grants 9603486, 9722606, and 0072204. The Polish groups were partially supported by KBN grant 2 P03B 04916 . The NCU group was partially supported by the NSC of Taiwan under contract NSC 89-2112-M-0088-024.

\section{REFERENCES}

1. Bjorken, J. D., Phys. Rev. D27, 140-151 (1983).

2. Back, B. B. et al., Phys. Rev. Lett., (in press) and hepex/0007036.

3. G. S. F. Stephans, "How Strange is PHOBOS" in Proceedings of the Vth International Conference on Strangeness in Quark Matter, to be published in Journal of Physics $G$.

4. Gyulassy, M., and Wang, X.-N., Phys. Rev. D44, 3501 (1991).

5. Wang X.N, and Gyulassy, M., Phys. Rev. Lett. (submitted) and nucl-th/0008014.

6. Werner, K, et al., Phys. Rep. 232, 87 (1993).

7. Bachler, J. et al., Nucl. Phys. A661, 45 (1999).

8. Abe, F., et al., Phys. Rev. D41, 2330 (1990).

9. Bialas, A, Bleszynski, M. and Czyz, W., Nulcear. Phys. B111, 461 (1976).

10. Eskola, K. J., et al., Nucl. Phys. B570, 379 (2000). 